\title{
Progressively Type-II Right Censored Order Statistics from Hjorth Distribution and Related Inference
}

\author{
Narinder Pushkarna, Jagdish Saran, Kanika Verma* \\ Department of Statistics, Faculty of Mathematical Sciences, University of Delhi, India
}

\begin{abstract}
In this paper some recurrence relations satisfied by single and product moments of progressively Type-II right censored order statistics from Hjorth distribution have been obtained. Then we use these results to compute the moments for all sample sizes and all censoring schemes $\left(R_{1}, R_{2}, \ldots, R_{m}\right), m \leq n$, which allow us to obtain BLUEs of location and scale parameters based on progressively Type-II right censored samples. The best linear unbiased predictors of censored failure times are then discussed briefly. Finally, a numerical example with real data is presented to illustrate the inferential method developed here.
\end{abstract}

Keywords Progressively Type-II censored order statistics, Hjorth distribution, single moments, product moments, recurrence relations, best linear unbiased estimators, best linear unbiased predictors.

\section{AMS 2010 subject classifications 62G30}

DOI: $10.19139 /$ soic-2310-5070-751

\section{Introduction}

The scheme of progressive Type-II censoring is of importance in reliability and life-testing experiments. It allows the experimenter to remove units from a life test at various stages during the experiment which may lead to a saving of costs and of time (see Cohen [12] and Sen [29]). In such a random experiment, a group of $\mathrm{n}$ independent and identical experimental units is put on a life test at time zero with continuous, identically distributed failure times $X_{1}, X_{2}, \ldots, X_{n}$. After the $j^{\text {th }}$ failure, a prespecified number $R_{j} \geq 0$ of the $n-j-\sum_{i=0}^{j-1} R_{i}$ remaining (or surviving) units are randomly withdrawn from the experiment, $1 \leq j \leq m, m \leq n, R_{0}=0$. Removed units thus become right censored at the time of failure of other units. This progressive censoring leads to $\mathrm{m}$ ordered observed failure times denoted by $X_{1: m: n}^{\left(R_{1}, R_{2}, \ldots, R_{m}\right)}, X_{2: m: n}^{\left(R_{1}, R_{2}, \ldots, R_{m}\right)}, \ldots, X_{m: m: n}^{\left(R_{1}, R_{2}, \ldots, R_{m}\right)}$, and these are called progressively Type-II right censored order statistics of size $\mathrm{m}$ from a sample of size $\mathrm{n}$ with progressive censoring scheme $\left(R_{1}, R_{2}, \ldots, R_{m}\right)$. Thus, in this type of sampling, $\mathrm{m}$ failures are observed, $\sum_{j=1}^{m} R_{j}$ units are progressively censored and $n=m+\sum_{j=1}^{m} R_{j}$ denotes the number of units in the life test. The withdrawal of units may be seen as a model describing drop-outs of units due to failures which have causes other than the specific one under study. In this sense, progressive censoring schemes are applied in clinical trials as well. Here, the drop-outs of patients may be caused by migration, lack of interest or by personal or ethical decisions, and they are regarded as random withdrawals. For a detailed discussion of progressive censoring and the relevant developments in this area, one may refer to Sen [29] and Balakrishnan and Aggarwala [4].

The situation with no censoring corresponds to the special case with $m=n$ and $R_{1}=R_{2}=\ldots=R_{m}=0$, whereas the situation with ordinary Type-II right censoring at a given order statistic corresponds to the special case with

\footnotetext{
${ }^{*}$ Correspondence to: Kanika verma (Email: kanikaverma29apr@gmail.com). Department of Statistics, University of Delhi, Delhi - 110007,
} India.

ISSN 2310-5070 (online) ISSN 2311-004X (print)

Copyright (C) 2020 International Academic Press 
$m<n, R_{1}=R_{2}=\ldots=R_{m-1}=0$ and $R_{m}=n-m$.

If the failure times of the $\mathrm{n}$ items originally on test are from a continuous population with c.d.f. $\mathrm{F}(\mathrm{x})$ and p.d.f. $\mathrm{f}(\mathrm{x})$, then the joint p.d.f. of $X_{1: m: n}^{\left(R_{1}, R_{2}, \ldots, R_{m}\right)}, X_{2: m: n}^{\left(R_{1}, R_{2}, \ldots, R_{m}\right)}, \ldots, X_{m: m: n}^{\left(R_{1}, R_{2}, \ldots, R_{m}\right)}$ is given by (cf. Balakrishnan and Sandhu [11] and Saran and Pushkarna [27])

$$
f_{X_{1: m: n}, \ldots, X_{m: m: n}}\left(x_{1}, x_{2}, \ldots, x_{m}\right)=A(n, m-1) \prod_{i=1}^{m} f\left(x_{i}\right)\left[1-F\left(x_{i}\right)\right]^{R_{i}}, 0 \leq x_{1}<x_{2}<\ldots<x_{m}<\infty,
$$

where $A(n, m-1)=n\left(n-R_{1}-1\right)\left(n-R_{1}-R_{2}-2\right) \ldots\left(n-R_{1}-R_{2}-\ldots-R_{m-1}-m+1\right)$.

Here, note that all the factors in $A(n, m-1)$ are positive integers. Also it may be observed that the different factors in $A(n, m-1)$ represent the number of units still on test immediately preceding the first, second, $\ldots, m^{\text {th }}$ observed failures, respectively. Similarly, for convenience in notation, let us define

$$
A(p, q)=p\left(p-R_{1}-1\right)\left(p-R_{1}-R_{2}-2\right) \ldots\left(p-R_{1}-R_{2}-\ldots-R_{q}-q\right),
$$

for $q=0,1, \ldots, p-1$, with all the factors being positive integers.

Progressive censoring and associated inferential procedures have been extensively studied in the literature for a number of distributions by several authors. Cohen ([12], [13], [14], [15] and [16]), Mann ([21], [22]), Cohen and Whitten [17], Viveros and Balakrishnan [30], Balakrishnan and Sandhu [11], Aggarwala and Balakrishnan [1] and Balakrishnan and Aggarwala [4] have derived recurrence relations for single and product moments of progressively Type-II right censored order statistics from exponential, Pareto and power function distributions and their truncated forms.

Saran and Pande [26], Saran and Pushkarna ([27], [28]), Saran et al. [25] and Pushkarna et al. [24] have derived recurrence relations for single and product moments of the corresponding progressively Type-II right censored order statistics from half logistic, Burr, left truncated logistic, Frechet and a general class of doubly truncated continuous distributions.

Mahmoud et al. [20] derived some new recurrence relations for single and product moments of progressively Type-II right censored order statistics from the linear exponential distribution and also obtained maximum likelihood estimators (MLEs) of the location and scale parameters. Balakrishnan et al. [5] and Balakrishnan and Saleh ([7], [8], [9], [10]) have established several recurrence relations for single and product moments of progressively Type-II right censored order statistics from logistic, half-logistic, log-logistic, generalized half logistic and generalized logistic distributions and utilized them to derive the best linear unbiased estimators of the location and scale parameters.

In this paper, we derive some recurrence relations satisfied by the single and product moments of progressively Type-II right censored order statistics from Hjorth distribution. These relations enable the recursive computation of moments for all sample sizes and all possible progressive censoring schemes. They generalize the corresponding results for exponential distribution due to Aggarwala and Balakrishnan [1]. Then we use these results to compute the means, variances and covariances of progressively Type-II right censored order statistics for some specific values of the parameters, which will be utilized to derive the best linear unbiased estimators (BLUEs) of location and scale parameters of the location-scale Hjorth distribution as well as their variances and covariances. Tables of these quantities are presented for different sample sizes up to $\mathrm{n}=8$ and some selected progressive censoring schemes, corresponding to particular values of the parameters. Further, for the special case $R_{1}=R_{2}=\ldots=R_{m}=0$, the derived results would reduce to the general recurrence relations for the usual order statistics from the Hjorth distribution. Also, we briefly discuss the best linear unbiased predictors (BLUPs) of the censored failure times by making use of the results developed on the BLUEs. Finally, one numerical example on real data is presented to illustrate all the methods of inference developed here.

\section{Hjorth distribution}

Hjorth distribution is a reliability distribution with increasing, decreasing, constant and bathtub shaped failure rates as its special cases. This distribution is also known as IDB distribution (cf. Hjorth [19]). Its p.d.f. $f(x)$, c.d.f. F(x) 


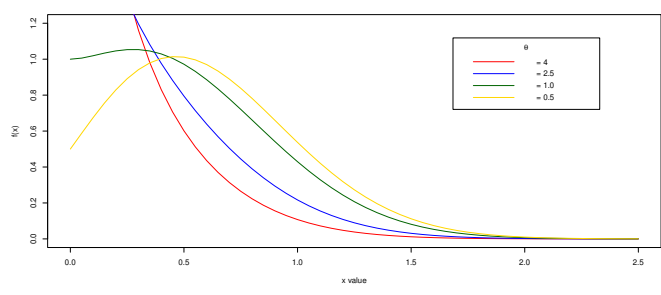

(a) p.d.f. of Hjorth distribution

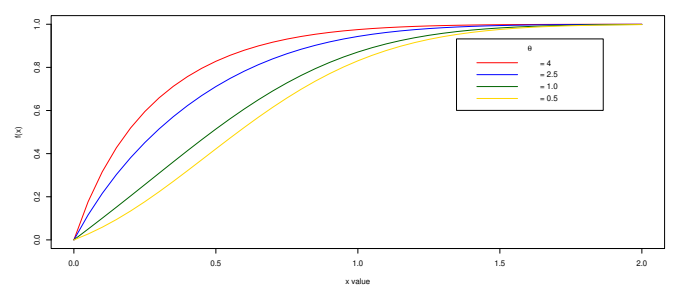

(b) c.d.f. of Hjorth distribution

Figure 2.1

and characterizing differential equation, respectively, are given by:

$$
\begin{gathered}
f(x)=\frac{[(1+\beta x) \delta x+\theta] e^{-\frac{\delta x^{2}}{2}}}{(1+\beta x)^{1+\frac{\theta}{\beta}}}, \quad x \geq 0, \quad \beta, \delta, \theta>0, \\
F(x)=1-\frac{e^{-\delta x^{2} / 2}}{(1+\beta x)^{\frac{\theta}{\beta}}}, \\
(1+\beta x) f(x)=[(1+\beta x) \delta x+\theta](1-F(x)) .
\end{gathered}
$$

The failure rate of this distribution is readily seen to be

$$
z(t)=\delta t+\frac{\theta}{1+\beta t} .
$$

Special cases of the Hjorth distribution are:

$$
\begin{aligned}
& \theta=0: \text { the Rayleigh distribution (a Weibull distribution), } \\
& \delta=\beta=0: \text { the exponential distribution (a Weibull distribution), } \\
& \delta=0: \text { decreasing failure rate, } \\
& \delta \geq \theta \beta: \text { increasing failure rate, } \\
& 0<\delta \leq \theta \beta: \text { bathtub curve. }
\end{aligned}
$$

More details on this distribution can be found in Hjorth [19]. The graphs of the p.d.f. and c.d.f. of Hjorth distribution as given in (2) and (3) for $\beta=2, \delta=3$ and for different values of $\theta=4.0,2.5,1.0$ and 0.50 are shown in Figures 2.1(a) and 2.1(b), respectively.

The c.d.f. of the location-scale parameter Hjorth distribution is given by

$$
F(x)=1-\frac{e^{-\delta\left(\frac{x-\mu}{\sigma}\right)^{2} / 2}}{\left(1+\beta\left(\frac{x-\mu}{\sigma}\right)\right)^{\frac{\theta}{\beta}}}, x \geq \mu, \mu \geq 0, \beta, \delta, \theta, \sigma>0 .
$$

\section{Recurrence relations for single moments}

In this section, we shall establish several recurrence relations for single moments of progressively Type-II right censored order statistics from Hjorth distribution satisfying the characterizing differential equation (4). Using (1), we have

$$
\begin{aligned}
\mu_{r: m: n}^{\left(R_{1}, R_{2}, \ldots, R_{m}\right)^{(k)}} & =E\left[X_{r: m: n}^{\left(R_{1}, R_{2}, \ldots, R_{m}\right)}\right]^{k} \\
& =A(n, m-1) \iint_{0 \leq x_{1}<x_{2}<\ldots<x_{m}<\infty} \ldots \int_{t=1} \int x_{r}^{k} \prod_{t=1}^{m} f\left(x_{t}\right)\left[1-F\left(x_{t}\right)\right]^{R_{t}} d x_{t} .
\end{aligned}
$$




\section{Theorem 1}

For $k \geq 0$,

$$
\mu_{1: 1: 1}^{(0)}{ }^{(k+3)}=\frac{k+3}{\delta \beta}\left[\mu_{1: 1: 1}^{(0)}{ }^{(k)}+\left(\beta-\frac{\theta}{k+1}\right) \mu_{1: 1: 1}^{(0)}{ }^{(k+1)}-\frac{\delta}{k+2} \mu_{1: 1: 1}{ }^{(0)}{ }^{(k+2)}\right]
$$

Proof

From (7), for $n=m=r=1$, we obtain

$$
\mu_{1: 1: 1}^{(0)}{ }^{(k)}+\beta \mu_{1: 1: 1}^{(0)}{ }^{(k+1)}=A(1,0) \int_{x_{1}} x_{1}{ }^{k}\left(1+\beta x_{1}\right) f\left(x_{1}\right) d x_{1},
$$

using (4), we have

$$
\begin{aligned}
\mu_{1: 1: 1}^{(0)}{ }^{(k)}+\beta \mu_{1: 1: 1}^{(0)}{ }^{(k+1)}= & \int_{x_{1}} x_{1}{ }^{k}\left(\delta x_{1}+\beta \delta x_{1}^{2}+\theta\right)\left(1-F\left(x_{1}\right)\right) d x_{1} \\
= & \delta \int_{x_{1}} x_{1}{ }^{k+1}\left(1-F\left(x_{1}\right)\right) d x_{1} \\
& +\delta \beta \int_{x_{1}} x_{1}^{k+2}\left(1-F\left(x_{1}\right)\right) d x_{1} \\
& +\theta \int_{x_{1}} x_{1}^{k}\left(1-F\left(x_{1}\right)\right) d x_{1} .
\end{aligned}
$$

Integrating by parts all integrals on the R.H.S. of the above equation by taking $\left(1-F\left(x_{1}\right)\right)$ for differentiation and the rest of the integrand for integration, and then after some simplification, it leads to the required result (8).

\section{Theorem 2}

For $n \geq 2$ and $k \geq 0$,

$$
\mu_{1: 1: n}^{(n-1)}{ }^{(k+3)}=\frac{k+3}{\delta \beta}\left[\frac{1}{n} \mu_{1: 1: n}^{(n-1)}{ }^{(k)}+\left(\frac{\beta}{n}-\frac{\theta}{k+1}\right) \mu_{1: 1: n}^{(n-1)}{ }^{(k+1)}-\frac{\delta}{k+2} \mu_{1: 1: n}^{(n-1)^{(k+2)}}\right] .
$$

\section{Proof}

Proceeding in a similar manner as in Theorem 1, we can easily establish the relation (9).

\section{Theorem 3}

For $2 \leq m \leq n-1, k \geq 0$ and $R_{1} \geq 0$,

$$
\begin{aligned}
\left.\mu_{1: m: n}^{\left(R_{1}, R_{2}, \ldots, R_{m}\right)}\right)^{(k)}+\beta \mu_{1: m: n}^{\left(R_{1}, R_{2}, \ldots, R_{m}\right)}{ }^{(k+1)}= & \theta\left[\frac{\left(n-R_{1}-1\right)}{k+1} \mu_{1: m-1: n}^{\left(R_{1}+R_{2}+1, R_{3}, \ldots, R_{m}\right)}{ }^{(k+1)}\right. \\
& \left.+\frac{\left(R_{1}+1\right)}{k+1} \mu_{1: m: n}^{\left(R_{1}, R_{2}, \ldots, R_{m}\right)}{ }^{(k+1)}\right] \\
+ & \delta\left[\frac{\left(n-R_{1}-1\right)}{k+2} \mu_{1: m-1: n}^{\left(R_{1}+R_{2}+1, R_{3}, \ldots, R_{m}\right)}\right)^{(k+2)} \\
& +\frac{\left(R_{1}+1\right)}{k+2} \mu_{1: m: n}^{\left.\left(R_{1}, R_{2}, \ldots, R_{m}\right)^{(k+2)}\right]} \\
+ & \delta \beta\left[\frac{\left(n-R_{1}-1\right)}{k+3} \mu_{1: m-1: n}^{\left(R_{1}+R_{2}+1, R_{3}, \ldots, R_{m}\right)^{(k+3)}}\right. \\
& \left.+\frac{\left(R_{1}+1\right)}{k+3} \mu_{1: m: n}^{\left(R_{1}, R_{2}, \ldots, R_{m}\right)}{ }^{(k+3)}\right]
\end{aligned}
$$


Proof

The relation in (10) may be proved by following exactly the same steps as those used in proving Theorem 4, which is presented next.

\section{Theorem 4}

For $2 \leq r \leq n-1, m<n, k \geq 0$ and $R_{r} \geq 0$,

$$
\begin{aligned}
& \mu_{r: m: n}^{\left(R_{1}, R_{2}, \ldots, R_{m}\right)}{ }^{(k)}+\beta \mu_{r: m: n}^{\left(R_{1}, R_{2}, \ldots, R_{m}\right)}{ }^{(k+1)}=\theta\left[\frac{\left(n-S_{r}-r\right)}{k+1} \mu_{r: m-1: n}^{\left(R_{1}, R_{2}, \ldots, R_{r-1}, R_{r}+R_{r+1}+1, R_{r+2}, \ldots, R_{m}\right)}{ }^{(k+1)}\right. \\
& -\frac{\left(n-S_{r-1}-r+1\right)}{k+1} \mu_{r-1: m-1: n}^{\left(R_{1}, R_{2}, \ldots, R_{r-2}, R_{r-1}+R_{r}+1, R_{r+1}, \ldots, R_{m}\right)}{ }^{(k+1)} \\
& +\frac{\left(R_{r}+1\right)}{k+1} \mu_{r: m: n}^{\left.\left(R_{1}, R_{2}, \ldots, R_{r}, \ldots, R_{m}\right)^{(k+1)}\right]} \\
& +\delta\left[\frac{\left(n-S_{r}-r\right)}{k+2} \mu_{r: m-1: n}^{\left(R_{1}, R_{2}, \ldots, R_{r-1}, R_{r}+R_{r+1}+1, R_{r+2}, \ldots, R_{m}\right)}{ }^{(k+2)}\right. \\
& -\frac{\left(n-S_{r-1}-r+1\right)}{k+2} \mu_{r-1: m-1: n}^{\left(R_{1}, R_{2}, \ldots, R_{r-2}, R_{r-1}+R_{r}+1, R_{r+1}, \ldots, R_{m}\right)}{ }^{(k+2)} \\
& +\frac{\left(R_{r}+1\right)}{k+2} \mu_{r: m: n}^{\left.\left(R_{1}, R_{2}, \ldots, R_{r}, \ldots, R_{m}\right)^{(k+2)}\right]} \\
& +\delta \beta\left[\frac{\left(n-S_{r}-r\right)}{k+3} \mu_{r: m-1: n}^{\left(R_{1}, R_{2}, \ldots, R_{r}+R_{r+1}+1, R_{r+2}, \ldots, R_{m}\right)}{ }^{(k+3)}\right. \\
& -\frac{\left(n-S_{r-1}-r+1\right)}{k+3} \mu_{r-1: m-1: n}^{\left(R_{1}, R_{2}, \ldots, R_{r-1}, R_{r-2}, R_{r-1}+R_{r}+1, R_{r+1}, \ldots, R_{m}\right)}{ }^{(k+3)} \\
& +\frac{\left(R_{r}+1\right)}{k+3} \mu_{r: m: n}^{\left.\left(R_{1}, R_{2}, \ldots, R_{r}, \ldots, R_{m}\right)^{(k+3)}\right],}
\end{aligned}
$$

where $S_{i}=R_{1}+R_{2}+\ldots+R_{i}, \quad 1 \leq i \leq m$.

\section{Proof}

Using (7) and (4), we have

$$
\begin{aligned}
& \mu_{r: m: n}^{\left(R_{1}, R_{2}, \ldots, R_{m}\right)^{(k)}}+\beta \mu_{r: m: n}^{\left(R_{1}, R_{2}, \ldots, R_{m}\right)}{ }^{(k+1)} \\
& =A(n, m-1) \int_{0 \leq x_{1}<x_{2}<\ldots<x_{r-1}<x_{r+1}<\ldots<x_{m}<\infty} I\left(x_{r-1}, x_{r+1}\right) \prod_{u=1, u \neq r}^{m}\left[1-F\left(x_{u}\right)\right]^{R_{u}} f\left(x_{u}\right) d x_{u},
\end{aligned}
$$

where

$$
I\left(x_{r-1}, x_{r+1}\right)=\int_{x_{r-1}}^{x_{r+1}} x_{r}^{k}\left(1+\beta x_{r}\right)\left[1-F\left(x_{r}\right)\right]^{R_{r}} f\left(x_{r}\right) d x_{r} .
$$


Using the characterizing differential equation (4), we have

$$
\begin{aligned}
I\left(x_{r-1}, x_{r+1}\right)= & \int_{x_{r-1}}^{x_{r+1}} x_{r}^{k}\left(\delta x_{r}+\delta \beta x_{r}^{2}+\theta\right)\left[1-F\left(x_{r}\right)\right]^{R_{r}+1} d x_{r} \\
= & \delta \int_{x_{r-1}}^{x_{r+1}} x_{r}^{k+1}\left[1-F\left(x_{r}\right)\right]^{R_{r}+1} d x_{r}+\delta \beta \int_{x_{r-1}}^{x_{r+1}} x_{r}^{k+2}\left[1-F\left(x_{r}\right)\right]^{R_{r}+1} d x_{r} \\
& \quad+\theta \int_{x_{r-1}}^{x_{r+1}} x_{r}^{k}\left[1-F\left(x_{r}\right)\right]^{R_{r}+1} d x_{r} \\
= & \delta I_{1}\left(x_{r-1}, x_{r+1}\right)+\delta \beta I_{2}\left(x_{r-1}, x_{r+1}\right)+\theta I_{0}\left(x_{r-1}, x_{r+1}\right),
\end{aligned}
$$

where

$$
I_{a}\left(x_{r-1}, x_{r+1}\right)=\int_{x_{r-1}}^{x_{r+1}} x_{r}^{k+a}\left[1-F\left(x_{r}\right)\right]^{R_{r}+1} d x_{r} ; a=0,1,2 .
$$

Integration by parts yields,

$$
\begin{aligned}
I_{a}\left(x_{r-1}, x_{r+1}\right)= & \frac{x_{r}^{k+a+1}}{k+a+1}\left[1-F\left(x_{r}\right)\right]^{R_{r}+1}||_{x_{r-1}}^{x_{r+1}}-\int\left(R_{r}+1\right)\left[1-F\left(x_{r}\right)\right]^{R_{r}}\left(-f\left(x_{r}\right)\right) \frac{x_{r}^{k+a+1}}{k+a+1} d x_{r} \\
= & \frac{1}{(k+a+1)}\left[x_{r+1}^{k+a+1}\left[1-F\left(x_{r+1}\right)\right]^{R_{r}+1}-x_{r-1}^{k+a+1}\left[1-F\left(x_{r-1}\right)\right]^{R_{r}+1}\right. \\
& \left.\quad+\left(R_{r}+1\right) \int\left[1-F\left(x_{r}\right)\right]^{R_{r}} x_{k+a+1} f\left(x_{r}\right) d x_{r}\right] .
\end{aligned}
$$

Upon substituting for $I_{0}\left(x_{r-1}, x_{r+1}\right), I_{1}\left(x_{r-1}, x_{r+1}\right)$ and $I_{2}\left(x_{r-1}, x_{r+1}\right)$ from equation (15) in (14) and then substituting the resultant expression for $I\left(x_{r-1}, x_{r+1}\right)$ in (12) and simplifying, it leads to Theorem 4.

Next, we state another result on single moments which can easily be established on similar lines.

\section{Theorem 5}

For $2 \leq m \leq n, k \geq 0$ and $R_{m} \geq 0$,

$$
\begin{aligned}
&\left.\mu_{m: m: n}^{\left(R_{1}, R_{2}, \ldots, R_{m}\right)}\right)^{(k)}+\beta \mu_{m: m: n}^{\left(R_{1}, R_{2}, \ldots, R_{m}\right)^{(k+1)}}=\theta\left[-\frac{\left(n-S_{m-1}-m+1\right)}{k+1} \mu_{m-1: m-1: n}^{\left(R_{1}, R_{2}, \ldots, R_{m-2}, R_{m-1}+R_{m}+1\right)}(k+1)\right. \\
&+\left.\frac{\left(R_{m}+1\right)}{k+1} \mu_{m: m: n}^{\left(R_{1}, R_{2}, \ldots, R_{m}\right)}(k+1)\right] \\
&+\delta\left[-\frac{\left(n-S_{m-1}-m+1\right)}{k+2} \mu_{m-1: m-1: n}^{\left(R_{1}, R_{2}, \ldots, R_{m-2}, R_{m-1}+R_{m}+1\right)}\right)^{(k+2)} \\
&\left.+\frac{\left(R_{m}+1\right)}{k+2} \mu_{m: m: n}^{\left(R_{1}, R_{2}, \ldots, R_{m}\right)}(k+2)\right] \\
&+\delta \beta\left[-\frac{\left(n-S_{m-1}-m+1\right)}{k+3} \mu_{m-1: m-1: n}^{\left(R_{1}, R_{2}, \ldots, R_{m-2}, R_{m-1}+R_{m}+1\right)}(k+3)\right. \\
&\left.+\frac{\left(R_{m}+1\right)}{k+3} \mu_{m: m: n}^{\left(R_{1}, R_{2}, \ldots, R_{m}\right)}{ }^{(k+3)}\right] .
\end{aligned}
$$

Remark 1. It may be mentioned that if $R_{1}=R_{2}=\ldots=R_{k-1}=0$, i.e. there is no censoring before the time of the $k^{t h}$ failure, then the first $\mathrm{k}$ progressively Type-II right censored order statistics are simply the first $\mathrm{k}$ usual 
order statistics. Thus, for the special case $R_{1}=R_{2}=\ldots=R_{m}=0$, so that $m=n$ in which case the progressively censored order statistics become the usual order statistics $X_{1: n}, X_{2: n}, \ldots, X_{n: n}$, the recurrence relations established in Section 3 would reduce to the corresponding recurrence relations for the single moments of usual order statistics from the Hjorth distribution satisfying the characterizing differential equation (4).

Remark 2. Setting $\delta=\beta=0$ and $\theta=1$, we observe that (4) reduces to $f(x)=1-F(x)$, which is the characterizing differential equation for the $\exp (1)$ distribution with p.d.f. $f(x)=e^{-x}, x>0$, the recurrence relations in Section 3 will reduce to and verify the corresponding recurrence relations established by Aggarwala and Balakrishnan [1] for the progressively Type-II right censored order statistics from exponential distribution. It may be mentioned that one can derive similar recurrence relations for progressively Type-II right censored order statistics by taking different values of parameters as special cases of Hjorth distribution as given in Section 2.

\section{Recurrence relations for product moments}

Using (1) we can write the product moments of progressively Type-II right censored order statistics as follows:

$$
\begin{aligned}
\mu_{r, s: m: n}^{\left(R_{1}, R_{2}, \ldots, R_{m}\right)^{\left(k_{1}, k_{2}\right)}} & =E\left[\left\{X_{r: m: n}^{\left(R_{1}, R_{2}, \ldots, R_{m}\right)}\right\}^{k_{1}}\left\{X_{s: m: n}^{\left(R_{1}, R_{2}, \ldots, R_{m}\right)}\right\}^{k_{2}}\right] \\
& =A(n, m-1) \int_{0 \leq x_{1}<x_{2}<\ldots<x_{m}<\infty} \int_{m} \ldots x_{r}^{k_{1}} x_{s}^{k_{2}} \prod_{t=1}^{m} f\left(x_{t}\right)\left[1-F\left(x_{t}\right)\right]^{R_{t}} d x_{t},
\end{aligned}
$$

where $1 \leq r<s \leq m \leq n$ and $k_{1}, k_{2} \geq 0$.

In this Section, we shall derive various recurrence relations for the product moments of progressively Type-II right censored order statistics from Hjorth distribution with p.d.f. $\mathrm{f}(\mathrm{x})$ and c.d.f. $\mathrm{F}(\mathrm{x})$ satisfying the characterizing differential equation (4).

\section{Theorem 6}

For $2 \leq s \leq m \leq n-R_{1}, k, t \geq 0$ and $R_{1} \geq 0$,

$$
\begin{aligned}
& \mu_{1, s: m: n}^{\left(R_{1}, R_{2}, \ldots, R_{m}\right)^{(k, t)}}+\beta \mu_{1, s: m: n}^{\left(R_{1}, R_{2}, \ldots, R_{m}\right)^{(k+1, t)}}=\frac{\theta}{k+1}\left[\left(n-R_{1}-1\right) \mu_{1, s-1: m-1: n}^{\left(R_{1}+R_{2}+1, R_{3}, R_{4}, \ldots, R_{m}\right)}{ }^{(k+1, t)}\right. \\
& \left.+\left(R_{1}+1\right) \mu_{1, s: m: n}^{\left(R_{1}, R_{2}, \ldots, R_{m}\right)^{(k+1, t)}}\right] \\
& +\frac{\delta}{k+2}\left[\left(n-R_{1}-1\right) \mu_{1, s-1: m-1: n}^{\left(R_{1}+R_{2}+1, R_{3}, R_{4}, \ldots, R_{m}\right)^{(k+2, t)}}\right. \\
& \left.+\left(R_{1}+1\right) \mu_{1, s: m: n}^{\left(R_{1}, R_{2}, \ldots, R_{m}\right)}{ }^{(k+2, t)}\right] \\
& +\frac{\delta \beta}{k+3}\left[\left(n-R_{1}-1\right) \mu_{1, s-1: m-1: n}^{\left(R_{1}+R_{2}+1, R_{3}, R_{4}, \ldots, R_{m}\right)}{ }^{(k+3, t)}\right. \\
& +\left(R_{1}+1\right) \mu_{1, s: m: n}^{\left.\left(R_{1}, R_{2}, \ldots, R_{m}\right)^{(k+3, t)}\right] .}
\end{aligned}
$$

Proof

The relation in (18) may be proved by following exactly the same steps as those used in proving Theorem 7.

Theorem 7

For $2 \leq r<s \leq m<n, k, t \geq 0$ and $R_{r} \geq 0$, 


$$
\begin{aligned}
& \mu_{r, s: m: n}^{\left(R_{1}, R_{2}, \ldots, R_{m}\right)^{(k, t)}}+\beta \mu_{r, s: m: n}^{\left(R_{1}, R_{2}, \ldots, R_{m}\right)^{(k+1, t)}} \\
& =\frac{\theta}{(k+1)}\left[\left(n-S_{r}-r\right) \mu_{r, s-1: m-1: n}^{\left(R_{1}, R_{2}, \ldots, R_{r-1}, R_{r}+R_{r+1}+1, R_{r+2}, \ldots, R_{m}\right)}{ }^{(k+1, t)}\right. \\
& -\left(n-S_{r-1}-r+1\right) \mu_{r-1, s-1: m-1: n}^{\left(R_{1}, R_{2}, \ldots, R_{r-2}, R_{r-1}+R_{r}+1, R_{r+1}, \ldots, R_{m}\right)^{(k+1, t)}} \\
& +\left(R_{r}+1\right) \mu_{r, s: m: n}^{\left.\left(R_{1}, R_{2}, \ldots, R_{r}, \ldots, R_{m}\right)^{(k+1, t)}\right]} \\
& +\frac{\delta}{(k+2)}\left[\left(n-S_{r}-r\right) \mu_{r, s-1: m-1: n}^{\left(R_{1}, R_{2}, \ldots, R_{r-1}, R_{r}+R_{r+1}+1, R_{r+2}, \ldots, R_{m}\right)}{ }^{(k+2, t)}\right. \\
& -\left(n-S_{r-1}-r+1\right) \mu_{r-1, s-1: m-1: n}^{\left(R_{1}, R_{2}, \ldots, R_{r-2}, R_{r-1}+R_{r}+1, R_{r+1}, \ldots, R_{m}\right)^{(k+2, t)}} \\
& +\left(R_{r}+1\right) \mu_{r, s: m: n}^{\left.\left(R_{1}, R_{2}, \ldots, R_{r}, \ldots, R_{m}\right)^{(k+2, t)}\right]} \\
& +\frac{\delta \beta}{(k+3)}\left[\left(n-S_{r}-r\right) \mu_{r, s-1: m-1: n}^{\left(R_{1}, R_{2}, \ldots, R_{r-1}, R_{r}+R_{r+1}+1, R_{r+2}, \ldots, R_{m}\right)}{ }^{(k+3, t)}\right. \\
& -\left(n-S_{r-1}-r+1\right) \mu_{r-1, s-1: m-1: n}^{\left(R_{1}, R_{2}, \ldots, R_{r-2}, R_{r-1}+R_{r}+1, R_{r+1}, \ldots, R_{m}\right)^{(k+3, t)}} \\
& +\left(R_{r}+1\right) \mu_{r, s: m: n}^{\left.\left(R_{1}, R_{2}, \ldots, R_{r}, \ldots, R_{m}\right)^{(k+3, t)}\right] .}
\end{aligned}
$$

\section{Proof}

From (17), let us consider for $2 \leq r<s \leq m<n, k, t \geq 0$ and $R_{r} \geq 0$

$$
\begin{aligned}
& \mu_{r, s: m: n}^{\left(R_{1}, R_{2}, \ldots, R_{m}\right)^{(k, t)}}+\beta \mu_{r, s: m: n}^{\left(R_{1}, R_{2}, \ldots, R_{m}\right)}{ }^{(k+1, t)} \\
& =A(n, m-1) \int_{0 \leq x_{1}<x_{2}<\ldots<x_{r-1}<x_{r+1}<\ldots x_{m}<\infty} \int_{s}^{k} I\left(x_{r-1}, x_{r+1}\right) \prod_{u=1, u \neq r}^{m} f\left(x_{u}\right)\left[1-F\left(x_{u}\right)\right]^{R_{u}} d x_{u},
\end{aligned}
$$

where $I\left(x_{r-1}, x_{r+1}\right)$ is the same as given in equation (13), or equivalently, in equations (14) and (15). Now upon using (15) in (14) and then putting the value of $I\left(x_{r-1}, x_{r+1}\right)$, so obtained, into the equation (20) and then simplifying, it leads to (19). 


\section{Theorem 8}

For $1 \leq r<s<m<n, k, t \geq 0$ and $R_{s} \geq 0$,

$$
\begin{aligned}
& \mu_{r, s: m: n}^{\left(R_{1}, R_{2}, \ldots, R_{m}\right)^{(k, t)}}+\beta \mu_{r, s: m: n}^{\left(R_{1}, R_{2}, \ldots, R_{m}\right)^{(k, t+1)}}=\frac{\theta}{(t+1)}\left[\left(n-S_{s}-s\right) \mu_{r, s: m-1: n}^{\left(R_{1}, R_{2}, \ldots, R_{s-1}, R_{s}+R_{s+1}+1, R_{s+2}, \ldots, R_{m}\right)^{(k, t+1)}}\right. \\
& -\left(n-S_{s-1}-s+1\right) \mu_{r, s-1: m-1: n}^{\left(R_{1}, R_{2}, \ldots, R_{s-2}, R_{s-1}+R_{s}+1, R_{s+1}, \ldots, R_{m}\right)}{ }^{(k, t+1)} \\
& \left.+\left(R_{s}+1\right) \mu_{r, s: m: n}^{\left(R_{1}, R_{2}, \ldots, R_{m}\right)^{(k, t+1)}}\right] \\
& +\frac{\delta}{(t+2)}\left[\left(n-S_{s}-s\right) \mu_{r, s: m-1: n}^{\left(R_{1}, R_{2}, \ldots, R_{s-1}, R_{s}+R_{s+1}+1, R_{s+2}, \ldots, R_{m}\right)}{ }^{(k, t+2)}\right. \\
& -\left(n-S_{s-1}-s+1\right) \mu_{r, s-1: m-1: n}^{\left(R_{1}, R_{2}, \ldots, R_{s-2}, R_{s-1}+R_{s}+1, R_{s+1}, \ldots, R_{m}\right)^{(k, t+2)}} \\
& \left.+\left(R_{s}+1\right) \mu_{r, s: m: n}^{\left(R_{1}, R_{2}, \ldots, R_{m}\right)}{ }^{(k, t+2)}\right] \\
& +\frac{\delta \beta}{(t+3)}\left[\left(n-S_{s}-s\right) \mu_{r, s: m-1: n}^{\left(R_{1}, R_{2}, \ldots, R_{s}+R_{s+1}+1, R_{s+2}, \ldots, R_{m}\right)}{ }^{(k, t+3)}\right. \\
& -\left(n-S_{s-1}-s+1\right) \mu_{r, s-1: m-1: n}^{\left(R_{1}, R_{2}, \ldots, R_{s-2}, R_{s-1}+R_{s}+1, R_{s+1}, \ldots, R_{m}\right)}{ }^{(k, t+3)}
\end{aligned}
$$

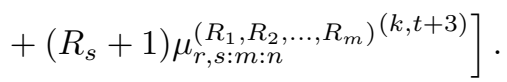

Proof

The relation in (21) may be proved by following the similar steps as those used in proving (19).

Next, we state another result on product moments which can easily be established on similar lines.

\section{Theorem 9}

For $1 \leq r<m<n, k, t \geq 0$ and $R_{m} \geq 0$,

$$
\begin{aligned}
& \mu_{r, m: m: n}^{\left(R_{1}, R_{2}, \ldots, R_{m}\right)}{ }^{(k, t)}+\beta \mu_{r, m: m: n}^{\left(R_{1}, R_{2}, \ldots, R_{m}\right)}{ }^{(k, t+1)} \\
& =\frac{\theta}{(t+1)}\left[-\left(n-S_{m-1}-m+1\right) \mu_{r, m-1: m-1: n}^{\left(R_{1}, R_{2}, \ldots, R_{m-2}, R_{m-1}+R_{m}+1\right)}(k, t+1)\right. \\
& \left.\left.\quad \quad+\left(R_{m}+1\right) \mu_{m: m: n}^{\left(R_{1}, R_{2}, \ldots, R_{m}\right)}\right)^{(k, t+1)}\right] \\
& \quad+\frac{\delta}{(t+2)}\left[-\left(n-S_{m-1}-m+1\right) \mu_{m-1: m-1: n}^{\left(R_{1}, R_{2}, \ldots, R_{m-2}, R_{m-1}+R_{m}+1\right)}(k, t+2)\right. \\
& \left.\left.\quad+\left(R_{m}+1\right) \mu_{m: m: n}^{\left(R_{1}, R_{2}, \ldots, R_{m}\right)}\right)^{(k, t+2)}\right] \\
& \quad+\frac{\delta \beta}{(t+3)}\left[-\left(n-S_{m-1}-m+1\right) \mu_{m-1: m-1: n}^{\left(R_{1}, R_{2}, \ldots, R_{m-2}, R_{m-1}+R_{m}+1\right)}(k, t+3)\right. \\
& \left.\quad+\left(R_{m}+1\right) \mu_{m: m: n}^{\left(R_{1}, R_{2}, \ldots, R_{m}\right)}{ }^{(k, t+3)}\right] .
\end{aligned}
$$

Remark 3. For the special case $R_{1}=R_{2}=\ldots=R_{m}=0$, the recurrence relations established in Section 4 reduce to the corresponding recurrence relations for the product moments of usual order statistics from the Hjorth distribution satisfying the characterizing differential equation (4).

Remark 4. Setting $\delta=\beta=0$ and $\theta=1$, we observe that (4) reduces to $f(x)=1-F(x)$, which is the characterizing differential equation for $\exp (1)$ distribution with p.d.f. $f(x)=e^{-x}, x>0$, the recurrence relations in Section 4 will reduce to and verify the corresponding recurrence relations established by Aggarwala and 
Balakrishnan [1] for the product moments of progressively Type-II right censored order statistics from exponential distribution.

It may be mentioned that one can derive similar recurrence relations for product moments of progressively Type-II right censored order statistics by taking different values of parameters as special cases of Hjorth distribution as given in Section 2.

\section{Numerical Results}

The recurrence relations obtained in the preceding Sections 3 and 4 allow us to evaluate the means, variances and covariances of progressively Type-II right censored order statistics from Hjorth distribution for all sample sizes ' $\mathrm{n}$ ' and all censoring schemes $\left(R_{1}, R_{2}, \ldots, R_{m}\right), m<n$. These quantities can be used for various inferential purposes; for example, they are useful in determining BLUEs of location/scale parameters and BLUPs of censored failure times. In this section, we compute means, variances and covariances of the progressively Type-II right censored order statistics from Hjorth distribution for some specific values of parameters, viz. $\beta=2, \delta=3, \theta=4$ for sample sizes up to 8 and for different choices of $\mathrm{m}$ and progressive censoring schemes $\left(R_{1}, R_{2}, \ldots, R_{m}\right), m<n$. These values are presented in the following Tables 1 and 2 .

Table 1. First four single moments of progressively Type-II right censored order statistics from Hjorth distribution

\begin{tabular}{|c|c|c|c|c|c|c|c|c|}
\hline S. No. & $\mathrm{m}$ & $\mathrm{n}$ & $\left(R_{1}, \ldots, R_{m}\right)$ & \multicolumn{2}{|c|}{$\mu_{r: m: n}^{\left(R_{1}, R_{2}, \ldots, R_{m}\right)^{(k)}}$} & \multicolumn{3}{|c|}{$r=1,2, \ldots, m$ and $k=1$} \\
\hline 1 & 2 & 4 & 1,1 & 0.066369 & 0.206697 & & & \\
\hline 2 & 2 & 5 & 1,2 & 0.052615 & 0.145046 & & & \\
\hline 3 & 2 & 5 & 2,1 & 0.052615 & 0.192479 & & & \\
\hline 4 & 2 & 5 & 0,3 & 0.052615 & 0.121387 & & & \\
\hline 5 & 2 & 5 & 3,0 & 0.052615 & 0.330690 & & & \\
\hline 6 & 2 & 6 & 2,2 & 0.043547 & 0.135628 & & & \\
\hline 7 & 2 & 7 & 3,2 & 0.037129 & 0.128931 & & & \\
\hline 8 & 3 & 4 & $0,0,1$ & 0.066369 & 0.159242 & 0.301608 & & \\
\hline 9 & 3 & 5 & $0,0,2$ & 0.052615 & 0.121387 & 0.216024 & & \\
\hline 10 & 3 & 5 & $0,1,1$ & 0.052615 & 0.121387 & 0.263571 & & \\
\hline 11 & 3 & 5 & $2,0,0$ & 0.052615 & 0.192479 & 0.468901 & & \\
\hline 12 & 3 & 6 & $1,1,1$ & 0.043547 & 0.112015 & 0.254038 & & \\
\hline 13 & 3 & 6 & $1,2,0$ & 0.043547 & 0.112014 & 0.391169 & & \\
\hline 14 & 3 & 6 & $1,0,2$ & 0.043547 & 0.112014 & 0.206469 & & \\
\hline 15 & 3 & 7 & $0,2,2$ & 0.037129 & 0.082054 & 0.175809 & & \\
\hline 16 & 3 & 7 & $1,2,1$ & 0.037129 & 0.091330 & 0.232938 & & \\
\hline 17 & 4 & 5 & $0,0,0,1$ & 0.052615 & 0.121387 & 0.216024 & 0.358664 & \\
\hline 18 & 4 & 5 & $1,0,0,0$ & 0.052615 & 0.145046 & 0.287344 & 0.559680 & \\
\hline 19 & 4 & 6 & $1,0,1,0$ & 0.043547 & 0.112015 & 0.206469 & 0.483520 & \\
\hline 20 & 4 & 7 & $1,2,0,0$ & 0.037129 & 0.091330 & 0.232938 & 0.508229 & \\
\hline 21 & 4 & 8 & $1,1,2,0$ & 0.032352 & 0.077131 & 0.146898 & 0.426065 & \\
\hline 22 & 4 & 8 & $1,3,0,0$ & 0.032352 & 0.077131 & 0.218325 & 0.494222 & \\
\hline 23 & 4 & 8 & $2,0,0,2$ & 0.032352 & 0.086387 & 0.156387 & 0.251806 & \\
\hline 24 & 5 & 6 & $0 * 4,1$ & 0.043547 & 0.097956 & 0.168248 & 0.263799 & 0.406097 \\
\hline 25 & 5 & 8 & $1 * 3,0 * 2$ & 0.032352 & 0.077131 & 0.146898 & 0.289752 & 0.562379 \\
\hline S. No. & $\mathrm{m}$ & $\mathrm{n}$ & $\left(R_{1}, \ldots, R_{m}\right)$ & & $\begin{array}{l}\left.\ell_{1}, R_{2}, \ldots, R_{m}\right) \\
m: n\end{array}$ & $, r=1,2$, & $m$ and $k$ & \\
\hline 1 & 2 & 4 & 1,1 & 0.009192 & 0.067926 & & & \\
\hline 2 & 2 & 5 & 1,2 & 0.005760 & 0.033252 & & & \\
\hline 3 & 2 & 5 & 2,1 & 0.005760 & 0.060425 & & & \\
\hline
\end{tabular}




\begin{tabular}{|c|c|c|c|c|c|c|c|c|}
\hline 4 & 2 & 5 & 0,3 & 0.005760 & 0.022921 & & & \\
\hline 5 & 2 & 5 & 3,0 & 0.005760 & 0.184343 & & & \\
\hline 6 & 2 & 6 & 2,2 & 0.003933 & 0.029581 & & & \\
\hline 7 & 2 & 7 & 3,2 & 0.002850 & 0.027187 & & & \\
\hline 8 & 3 & 4 & $0,0,1$ & 0.009192 & 0.039451 & 0.124874 & & \\
\hline 9 & 3 & 5 & $0,0,2$ & 0.005760 & 0.022921 & 0.064248 & & \\
\hline 10 & 3 & 5 & $0,1,1$ & 0.005760 & 0.022921 & 0.097929 & & \\
\hline 11 & 3 & 5 & $2,0,0$ & 0.005760 & 0.060425 & 0.308261 & & \\
\hline 12 & 3 & 6 & $1,1,1$ & 0.003933 & 0.019711 & 0.092033 & & \\
\hline 13 & 3 & 6 & $1,2,0$ & 0.003933 & 0.019711 & 0.230183 & & \\
\hline 14 & 3 & 6 & $1,0,2$ & 0.003933 & 0.019711 & 0.059191 & & \\
\hline 15 & 3 & 7 & $0,2,2$ & 0.002850 & 0.010429 & 0.043946 & & \\
\hline 16 & 3 & 7 & $1,2,1$ & 0.002850 & 0.013036 & 0.079381 & & \\
\hline 17 & 4 & 5 & $0,0,0,1$ & 0.005760 & 0.022921 & 0.064248 & 0.165292 & \\
\hline 18 & 4 & 5 & $1,0,0,0$ & 0.005760 & 0.033252 & 0.114770 & 0.405006 & \\
\hline 19 & 4 & 6 & $1,0,1,0$ & 0.003933 & 0.019711 & 0.059191 & 0.315679 & \\
\hline 20 & 4 & 7 & $1,2,0,0$ & 0.002850 & 0.013036 & 0.079381 & 0.346407 & \\
\hline 21 & 4 & 8 & $1,3,0,0$ & 0.000031 & 0.000322 & 0.017726 & 0.277668 & \\
\hline 22 & 4 & 8 & $2,0,0,2$ & 0.000030 & 0.000542 & 0.003307 & 0.015794 & \\
\hline 23 & 4 & 8 & $1,1,2,0$ & 0.000031 & 0.000322 & 0.002642 & 0.196049 & \\
\hline 24 & 5 & 6 & $0 * 4,1$ & 0.003933 & 0.014898 & 0.038967 & 0.089529 & 0.203174 \\
\hline 25 & 5 & 8 & $1 * 3,0 * 2$ & 0.002158 & 0.009257 & 0.030166 & 0.112655 & 0.404858 \\
\hline S. No. & $\mathrm{m}$ & $\mathrm{n}$ & $\left(R_{1}, \ldots, R_{m}\right)$ & $\mu_{r: m: n}^{\left(R_{1}, R_{2}\right.}$ &.,$\left.R_{m}\right)^{(k)}, r$ & $=1,2, \ldots, m$ & nd $k=3$ & \\
\hline 1 & 2 & 4 & 1,1 & 0.001959 & 0.030602 & & & \\
\hline 2 & 2 & 5 & 1,2 & 0.000973 & 0.010507 & & & \\
\hline 3 & 2 & 5 & 2,1 & 0.000973 & 0.026486 & & & \\
\hline 4 & 2 & 5 & 0,3 & 0.000973 & 0.005906 & & & \\
\hline 5 & 2 & 5 & 3,0 & 0.000973 & 0.141576 & & & \\
\hline 6 & 2 & 6 & 2,2 & 0.000548 & 0.009025 & & & \\
\hline 7 & 2 & 7 & 3,2 & 0.000337 & 0.008123 & & & \\
\hline 8 & 3 & 4 & $0,0,1$ & 0.001959 & 0.013268 & 0.065272 & & \\
\hline 9 & 3 & 5 & $0,0,2$ & 0.000973 & 0.005906 & 0.024311 & & \\
\hline 10 & 3 & 5 & $0,1,1$ & 0.000973 & 0.005906 & 0.047067 & & \\
\hline 11 & 3 & 5 & $2,0,0$ & 0.000973 & 0.026486 & 0.256666 & & \\
\hline 12 & 3 & 6 & $1,1,1$ & 0.000548 & 0.004782 & 0.043513 & & \\
\hline 13 & 3 & 6 & $1,2,0$ & 0.000548 & 0.004782 & 0.1797883 & & \\
\hline 14 & 3 & 6 & $1,0,2$ & 0.000548 & 0.004782 & 0.021753 & & \\
\hline 15 & 3 & 7 & $0,2,2$ & 0.000337 & 0.001812 & 0.014435 & & \\
\hline 16 & 3 & 7 & $1,2,1$ & 0.000337 & 0.002562 & 0.036056 & & \\
\hline 17 & 4 & 5 & $0,0,0,1$ & 0.000973 & 0.005906 & 0.024311 & 0.092579 & \\
\hline 18 & 4 & 5 & $1,0,0,0$ & 0.000973 & 0.010507 & 0.058445 & 0.355776 & \\
\hline 19 & 4 & 6 & $1,0,1,0$ & 0.000548 & 0.004782 & 0.021754 & 0.258806 & \\
\hline 20 & 4 & 7 & $1,2,0,0$ & 0.000337 & 0.002562 & 0.036056 & 0.293434 & \\
\hline 21 & 4 & 8 & $1,1,2,0$ & 0.002158 & 0.009257 & 0.030166 & 0.258757 & \\
\hline 22 & 4 & 8 & $1,3,0,0$ & 0.002158 & 0.009257 & 0.071410 & 0.331807 & \\
\hline 23 & 4 & 8 & $2,0,0,2$ & 0.002158 & 0.011764 & 0.034077 & 0.082350 & \\
\hline 24 & 5 & 6 & $0 * 4,1$ & 0.000548 & 0.003097 & 0.011523 & 0.037099 & 0.120319 \\
\hline 25 & 5 & 8 & $1 * 3,0 * 2$ & 0.000221 & 0.001527 & 0.008038 & 0.055338 & 0.353311 \\
\hline S. No. & $\mathrm{m}$ & $\mathrm{n}$ & $\left(R_{1}, \ldots, R_{m}\right)$ & $\mu_{r: m: n}^{\left(R_{1}, R_{2}\right.}$ & $\left.R_{m}\right)^{(k)}$ & 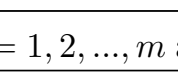 & dd $k=4$ & \\
\hline
\end{tabular}




\begin{tabular}{ccccccccc}
2 & 2 & 5 & 1,2 & 0.000223 & 0.004222 & & & \\
3 & 2 & 5 & 2,1 & 0.000223 & 0.014770 & & & \\
4 & 2 & 5 & 0,3 & 0.000223 & 0.001925 & & \\
5 & 2 & 5 & 3,0 & 0.000223 & 0.134627 & & & \\
6 & 2 & 6 & 2,2 & 0.000104 & 0.003541 & & & \\
7 & 2 & 7 & 3,2 & 0.000054 & 0.003148 & & & \\
8 & 3 & 4 & $0,0,1$ & 0.000563 & 0.005600 & 0.040819 & & \\
9 & 3 & 5 & $0,0,2$ & 0.000223 & 0.001925 & 0.011112 & & \\
10 & 3 & 5 & $0,1,1$ & 0.000223 & 0.001925 & 0.027615 & & \\
11 & 3 & 5 & $2,0,0$ & 0.000223 & 0.014770 & 0.254485 & & \\
12 & 3 & 6 & $1,1,1$ & 0.000104 & 0.001482 & 0.025268 & & \\
13 & 3 & 6 & $1,2,0$ & 0.000104 & 0.001482 & 0.171873 & & \\
14 & 3 & 6 & $1,0,2$ & 0.000104 & 0.001482 & 0.009717 & & \\
15 & 3 & 7 & $0,2,2$ & 0.000054 & 0.000401 & 0.005897 & & \\
16 & 3 & 7 & $1,2,1$ & 0.000054 & 0.000644 & 0.020421 & & \\
17 & 4 & 5 & $0,0,0,1$ & 0.000223 & 0.001925 & 0.011112 & 0.060623 & \\
18 & 4 & 5 & $1,0,0,0$ & 0.000223 & 0.004222 & 0.035867 & 0.363793 & \\
19 & 4 & 6 & $1,0,1,0$ & 0.000104 & 0.001482 & 0.009717 & 0.252951 & \\
20 & 4 & 7 & $1,2,0,0$ & 0.000054 & 0.000644 & 0.020421 & 0.29349 & \\
21 & 4 & 8 & $1,1,2,0$ & 0.000031 & 0.000322 & 0.017726 & 0.277668 & \\
22 & 4 & 8 & $1,3,0,0$ & 0.000030 & 0.000542 & 0.003307 & 0.015794 & \\
23 & 4 & 8 & $2,0,0,2$ & 0.000031 & 0.000322 & 0.002642 & 0.196049 & \\
24 & 5 & 6 & $0 * 4,1$ & 0.000104 & 0.000818 & 0.004139 & 0.018084 & 0.081892 \\
25 & 5 & 8 & $1 * 3,0 * 2$ & 0.000031 & 0.000322 & 0.002642 & 0.032810 & 0.359287 \\
\hline
\end{tabular}

Table 2. Variances and covariances of progressively Type-II right censored order statistics from Hjorth distribution

\begin{tabular}{cccccccccc}
\hline $\mathrm{m}$ & $\mathrm{s}$ & $\mathrm{r}$ & $\sigma_{r, s: m: 4}^{(1,1)}$ & $\sigma_{r, s: m: 5}^{(2,1)}$ & $\sigma_{r, s: m: 5}^{(1,2)}$ & $\sigma_{r, s: m: 5}^{(0,3)}$ & $\sigma_{r, s: m: 5}^{(3,0)}$ & $\sigma_{r, s: m: 6}^{(2,2)}$ & $\sigma_{r, s: m: 7}^{(3,2)}$ \\
\hline 2 & 1 & 1 & 0.004787 & 0.002992 & 0.002992 & 0.002992 & 0.002992 & 0.002036 & 0.001471 \\
& 2 & 1 & 0.004923 & 0.003106 & 0.003098 & 0.003085 & 0.003054 & 0.002120 & 0.001538 \\
& & 2 & 0.004923 & 0.003106 & 0.003098 & 0.003085 & 0.003054 & 0.002120 & 0.001538 \\
\hline $\mathrm{m}$ & $\mathrm{s}$ & $\mathrm{r}$ & $\sigma_{r, s: m: 4}^{(0,0,1)}$ & $\sigma_{r, s: m: 5}^{(0,0,2)}$ & $\sigma_{r, s: m: 5}^{(0,1,1)}$ & $\sigma_{r, s: m: 5}^{(2,0,0)}$ & $\sigma_{r, s: m: 6}^{(1,1,1)}$ & $\sigma_{r, s: m: 6}^{(1,2,0)}$ & $\sigma_{r, s: m: 6}^{(1,0,2)}$ \\
\hline 3 & 1 & 1 & 0.004787 & 0.002992 & 0.002992 & 0.002992 & 0.002036 & 0.002036 & 0.002036 \\
& 2 & 1 & 0.004921 & 0.003085 & 0.003085 & 0.003106 & 0.002108 & 0.002108 & 0.002108 \\
& & 2 & 0.014093 & 0.008186 & 0.008186 & 0.023377 & 0.007164 & 0.007164 & 0.007164 \\
& 3 & 1 & 0.004926 & 0.003137 & 0.003127 & 0.003001 & 0.002150 & 0.002097 & 0.002154 \\
& & 2 & 0.014066 & 0.008310 & 0.008280 & 0.022222 & 0.007269 & 0.007077 & 0.00729 \\
& & 3 & 0.033907 & 0.017581 & 0.028460 & 0.088392 & 0.027497 & 0.077169 & 0.016562 \\
\hline & $\mathrm{s}$ & $\mathrm{r}$ & $\sigma_{r, s: m: 7}^{(0,2,2)}$ & $\sigma_{r, s: m: 7}^{(1,2,1)}$ & & & & & \\
\cline { 2 - 10 } & 1 & 1 & 0.001471 & 0.001471 & & & & & \\
& 2 & 1 & 0.001515 & 0.001521 & & & & & \\
& & 2 & 0.003696 & 0.004694 & & & & & \\
& 3 & 1 & 0.001561 & 0.001564 & & & & & \\
& & 2 & 0.003805 & 0.004811 & & & & & \\
& & 3 & 0.013037 & 0.025120 & & & & & \\
\hline $\mathrm{m}$ & $\mathrm{s}$ & $\mathrm{r}$ & $\sigma_{r, s: m: 5}^{(0,0,0,1)}$ & $\sigma_{r, s: m: 5}^{(1,0,0,0)}$ & $\sigma_{r, s: m: 6}^{(1,0,1,0)}$ & $\sigma_{r, s: m: 7}^{(1,2,0,0)}$ & $\sigma_{r, s: m: 8}^{(1,3,0,0)}$ & $\sigma_{r, s: m: 8}^{(1,1,2,0)}$ & $\sigma_{r, s: m: 8}^{(2,0,0,2)}$ \\
\hline 4 & 1 & 1 & 0.002992 & 0.002992 & 0.002036 & 0.001471 & 0.001111 & 0.001111 & 0.001111 \\
& 2 & 1 & 0.003085 & 0.003098 & 0.002108 & 0.001521 & 0.001146 & 0.001146 & 0.001151 \\
& & 2 & 0.008186 & 0.012214 & 0.007164 & 0.004694 & 0.003308 & 0.003308 & 0.004301
\end{tabular}




\begin{tabular}{lllllllll}
3 & 1 & 0.003137 & 0.003122 & 0.002154 & 0.001564 & 0.001187 & 0.001180 & 0.001183 \\
& 2 & 0.008310 & 0.012234 & 0.007291 & 0.004811 & 0.003416 & 0.003400 & 0.004406 \\
& 3 & 0.017581 & 0.032203 & 0.016562 & 0.025120 & 0.023745 & 0.008587 & 0.009620 \\
4 & 1 & 0.003107 & 0.002941 & 0.002068 & 0.001498 & 0.001144 & 0.001160 & 0.001198 \\
& 2 & 0.008219 & 0.011465 & 0.006970 & 0.004594 & 0.003284 & 0.003333 & 0.004451 \\
& 3 & 0.017347 & 0.030049 & 0.015772 & 0.023660 & 0.022425 & 0.008369 & 0.009699 \\
& 4 & 0.036652 & 0.091765 & 0.081887 & 0.088111 & 0.087552 & 0.077225 & 0.018944 \\
\hline
\end{tabular}

\section{BLUEs of $\mu$ and $\sigma$}

Suppose we obtain a progressively Type-II censored data from the location-scale parameter Hjorth distribution with c.d.f. as given in (6).

In this section, we make use of means, variances and covariances of progressively Type-II right censored order statistics as determined by using the recurrence relations given in Sections 3 and 4 for deriving the BLUEs of the location and scale parameters $\mu$ and $\sigma$ as well as the variances and covariance of these estimates.

Let $Y_{1: m: n} \leq Y_{2: m: n} \leq \ldots \leq Y_{m: m: n}$ be a progressively Type-II right censored sample from the location-scale parameter Hjorth distribution (6), and let $X_{i: m: n}=\frac{\left(Y_{i: m: n}-\mu\right)}{\sigma}, i=1,2, \ldots, m$, be the corresponding progressively Type-II right censored order statistics from the location-scale parameter Hjorth distribution.

Let us denote $E\left(X_{i: m: n}\right)$ by $\mu_{i}, \operatorname{Var}\left(X_{i: m: n}\right)$ by $\sigma_{i, i}$ and $\operatorname{Cov}\left(X_{i: m: n}, X_{j: m: n}\right)$ by $\sigma_{i, j}$; furthermore, let

$$
\begin{gathered}
Y=\left(Y_{1: m: n}, Y_{2: m: n}, \ldots, Y_{m: m: n}\right)^{T}, \\
\mu=\left(\mu_{1}, \mu_{2}, \ldots, \mu_{m}\right)^{T}, \\
\mathbf{1}=(\underbrace{1,1, \ldots, 1})^{T}
\end{gathered}
$$

and

$$
\sum=\left(\sigma_{r, s: n}\right), 1 \leq r, s \leq m .
$$

Then, the BLUEs of $\mu$ and $\sigma$ are obtained by minimizing the generalized variance $Q(\delta)=(Y-A \delta)^{T} \sum^{T}(Y-$ $A \delta)$ with respect to $\delta$, where $\delta=(\mu, \sigma)^{T}$, A is $m \times 2$ matrix $(\mathbf{1}, \mu), \mathbf{1}$ is $m \times 1$ vector with components all 1's, $\mu$ is the mean vector of $\mathbf{X}$, and $\sum$ is the variance-covariance matrix of $\mathbf{X}$. The minimization leads to the expressions for the BLUES's of $\mu$ and $\sigma$ as (see Anrold et al. [2] and Balakrishnan and Cohen [6])

$$
\mu^{*}=\left\{\frac{\mu^{T} \sum^{-1} \mu \mathbf{1}^{T} \sum^{-1}-\mu^{T} \sum^{-1} \mathbf{1} \mu^{T} \sum^{-1}}{\left(\mu^{T} \sum^{-1} \mu\right)\left(\mathbf{1}^{T} \sum^{-1} \mathbf{1}\right)-\left(\mu^{T} \sum^{-1} \mathbf{1}\right)^{2}}\right\} Y=\sum_{r=1}^{m} a_{r} Y_{r: m: n}
$$

and

$$
\sigma^{*}=\left\{\frac{1^{T} \sum^{-1} \mathbf{1} \mu^{T} \sum^{-1}-\mathbf{1}^{T} \sum^{-1} \mu \mathbf{1}^{T} \sum^{-1}}{\left(\mu^{T} \sum^{-1} \mu\right)\left(\mathbf{1}^{T} \sum^{-1} \mathbf{1}\right)-\left(\mu^{T} \sum^{-1} \mathbf{1}\right)^{2}}\right\} Y=\sum_{r=1}^{m} b_{r} Y_{r: m: n},
$$

and the variances and covariance of these BLUEs are given by

$$
\begin{aligned}
& \operatorname{Var}\left(\mu^{*}\right)=\sigma^{2}\left\{\frac{\mu^{T} \sum^{-1} \mu}{\left(\mu^{T} \sum^{-1} \mu\right)\left(\mathbf{1}^{T} \sum^{-1} \mathbf{1}\right)-\left(\mu^{T} \sum^{-1} \mathbf{1}\right)^{2}}\right\}=\sigma^{2} V_{1}, \\
& \operatorname{Var}\left(\sigma^{*}\right)=\sigma^{2}\left\{\frac{\mathbf{1}^{T} \sum^{-1} \mathbf{1}}{\left(\mu^{T} \sum^{-1} \mu\right)\left(\mathbf{1}^{T} \sum^{-1} \mathbf{1}\right)-\left(\mu^{T} \sum^{-1} \mathbf{1}\right)^{2}}\right\}=\sigma^{2} V_{2}
\end{aligned}
$$


and

$$
\operatorname{Cov}\left(\mu^{*}, \sigma^{*}\right)=\sigma^{2}\left\{\frac{-\mu^{T} \sum^{-1} \mathbf{1}}{\left(\mu^{T} \sum^{-1} \mu\right)\left(\mathbf{1}^{T} \sum^{-1} \mathbf{1}\right)-\left(\mu^{T} \sum^{-1} \mathbf{1}\right)^{2}}\right\}=\sigma^{2} V_{3} .
$$

The coefficients of the BLUEs in (23) and (24) satisfy the conditions $\sum_{r=1}^{m} a_{r}=1$ and $\sum_{r=1}^{m} b_{r}=0$ respectively. The coefficients of the BLUEs for $\mu$ and $\sigma$, and variances and covariance of these estimates are presented in Tables 3,4 and 5, respectively, for various sample sizes up to $\mathrm{n}=8$ and for different choices of $\mathrm{m}$ and progressive censoring schemes.

Table 3. Coefficients of the BLUEs of location parameter

\begin{tabular}{|c|c|c|c|c|c|c|c|c|}
\hline S.No. & $m$ & $n$ & $\left(R_{1}, R_{2}, \ldots, R_{m}\right)$ & \multicolumn{5}{|c|}{$a_{i}, i=1,2, \ldots, m$} \\
\hline 1 & 2 & 4 & 1,1 & 1.472961 & -0.472961 & & & \\
\hline 2 & 2 & 5 & 1,2 & 1.569237 & -0.569237 & & & \\
\hline 3 & 2 & 5 & 2,1 & 1.376189 & -0.376189 & & & \\
\hline 4 & 2 & 5 & 0,3 & 1.765070 & -0.765070 & & & \\
\hline 5 & 2 & 5 & 3,0 & 1.189212 & -0.189212 & & & \\
\hline 6 & 2 & 6 & 2,2 & 1.472919 & -0.472919 & & & \\
\hline 7 & 2 & 7 & 3,2 & 1.404446 & -0.404446 & & & \\
\hline 8 & 3 & 4 & $0,0,1$ & 1.352880 & -0.116892 & -0.235988 & & \\
\hline 9 & 3 & 5 & $0,0,2$ & 1.381140 & -0.102141 & -0.278998 & & \\
\hline 10 & 3 & 5 & $0,1,1$ & 1.377761 & -0.190427 & -0.187334 & & \\
\hline 11 & 3 & 5 & $2,0,0$ & 1.178155 & -0.077954 & -0.100201 & & \\
\hline 12 & 3 & 6 & $1,2,0$ & 1.299918 & -0.217482 & -0.082436 & & \\
\hline 13 & 3 & 6 & $1,0,2$ & 1.316218 & -0.084399 & -0.231819 & & \\
\hline 14 & 3 & 6 & $1,1,1$ & 1.313292 & -0.157708 & -0.155584 & & \\
\hline 15 & 3 & 7 & $0,2,2$ & 1.413979 & -0.216323 & -0.197656 & & \\
\hline 16 & 3 & 7 & $1,2,1$ & 1.338522 & -0.205897 & -0.132625 & & \\
\hline 17 & 4 & 5 & $0,0,0,1$ & 1.252672 & -0.069726 & -0.057280 & -0.125666 & \\
\hline 18 & 4 & 5 & $1,0,0,0$ & 1.214988 & -0.130246 & -0.035790 & -0.048953 & \\
\hline 19 & 4 & 6 & $1,0,1,0$ & 1.202378 & -0.055281 & -0.090079 & -0.057017 & \\
\hline 20 & 4 & 7 & $1,2,0,0$ & 1.214988 & -0.130246 & -0.035790 & -0.048953 & \\
\hline 21 & 4 & 8 & $1,1,2,0$ & 1.231181 & -0.084092 & -0.105042 & -0.042047 & \\
\hline 22 & 4 & 8 & $1,3,0,0$ & 1.226968 & -0.1531338 & -0.03119558 & -0.04263828 & \\
\hline 23 & 4 & 8 & $2,0,0,2$ & 1.198464 & -0.04572552 & -0.03812323 & -0.1146151 & \\
\hline 24 & 5 & 6 & $0 * 4,1$ & 1.199748 & -0.047806 & -0.039169 & -0.033903 & -0.078870 \\
\hline 25 & 5 & 6 & $1 * 3,0 * 2$ & 1.172194 & -0.063154 & -0.052897 & -0.023079 & -0.033063 \\
\hline
\end{tabular}


Table 4. Coefficients of the BLUEs of scale parameter

\begin{tabular}{|c|c|c|c|c|c|c|c|c|}
\hline S.No. & $\mathrm{m}$ & $\mathrm{n}$ & $\left(R_{1}, R_{2}, \ldots, R_{m}\right)$ & \multicolumn{5}{|c|}{$b_{i}, i=1,2, \ldots, m$} \\
\hline 1 & 2 & 4 & 1,1 & -7.126182 & 7.126182 & & & \\
\hline 2 & 2 & 5 & 1,2 & -10.818890 & 10.818890 & & & \\
\hline 3 & 2 & 5 & 2,1 & -7.149825 & 7.149825 & & & \\
\hline 4 & 2 & 5 & 0,3 & -14.540870 & 14.540870 & & & \\
\hline 5 & 2 & 5 & 3,0 & -3.596154 & 3.596154 & & & \\
\hline 6 & 2 & 6 & 2,2 & -10.859990 & 10.859990 & & & \\
\hline 7 & 2 & 7 & 3,2 & -10.892980 & 10.892980 & & & \\
\hline 8 & 3 & 4 & $0,0,1$ & -5.204608 & 1.575683 & 3.628925 & & \\
\hline 9 & 3 & 5 & $0,0,2$ & -7.137124 & 1.756899 & 5.380225 & & \\
\hline 10 & 3 & 5 & $0,1,1$ & -7.037568 & 3.408359 & 3.629209 & & \\
\hline 11 & 3 & 5 & $2,0,0$ & -3.303420 & 1.357226 & 1.946194 & & \\
\hline 12 & 3 & 6 & $1,1,1$ & -7.069212 & 3.436102 & 3.633110 & & \\
\hline 13 & 3 & 6 & $1,2,0$ & -6.700802 & 4.762049 & 1.938753 & & \\
\hline 14 & 3 & 6 & $1,0,2$ & -7.167823 & 1.776460 & 5.391363 & & \\
\hline 15 & 8 & 0 & $0,2,2$ & -11.014400 & 5.626087 & 5.388309 & & \\
\hline 16 & 3 & 7 & $1,2,1$ & -8.971029 & 5.342983 & 3.628045 & & \\
\hline 17 & 4 & 5 & $0,0,0,1$ & -4.590840 & 1.114410 & 0.985681 & 2.490749 & \\
\hline 18 & 4 & 5 & $1,0,0,0$ & -5.498248 & 3.216274 & 0.905817 & 1.376158 & \\
\hline 19 & 4 & 6 & $1,0,1,0$ & -4.429882 & 1.076152 & 1.982419 & 1.371311 & \\
\hline 20 & 4 & 7 & $1,2,0,0$ & -5.498248 & 3.216274 & 0.905817 & 1.376158 & \\
\hline 21 & 4 & 8 & $1,1,2,0$ & -6.881663 & 2.382165 & 3.145720 & 1.353778 & \\
\hline 22 & 4 & 8 & $1,3,0,0$ & -6.686736 & 4.405297 & 0.9097395 & 1.371700 & \\
\hline 23 & 4 & 8 & $2,0,0,2$ & -5.978192 & 1.259320 & 1.085963 & 3.632910 & \\
\hline 24 & 5 & 6 & $0 * 4,1$ & -4.293554 & 0.882489 & 0.765931 & 0.720468 & 1.924666 \\
\hline 25 & 5 & 6 & $1 * 3,0 * 2$ & -4.978081 & 1.706113 & 1.500884 & 0.686065 & 1.085019 \\
\hline
\end{tabular}

\section{Best Linear Unbiased Predictors (BLUPs)}

Based on observations on $m$ progressively Type-II right censored order statistics $Y_{1: m: n}^{\left(R_{1}, \ldots, R_{m}\right)}, \ldots, Y_{m: m: n}^{\left(R_{1}, \ldots, R_{m}\right)}$, we discuss the prediction of times to failure of the last $R_{m}(\geq 1)$ units still surviving at the observation $Y_{m: m: n}^{\left(R_{1}, \ldots, R_{m}\right)}$. Of course, one can discuss the prediction of other censored failure times in a similar manner as well. Doganaksoy and Balakrishnan [18] established that the BLUEs remain unchanged if the BLUPs of future failures are treated as observed values.

The BLUP of $Y_{m+1: m+1: n}^{\left(R_{1}, \ldots, R_{m-1}, 0, R_{m}-1\right)}$ from any location-scale family of distributions is given by

$$
Y_{m+1: m+1: n}^{\left(R_{1}, \ldots, R_{m-1}, 0, R_{m}-1\right)^{*}}=\mu^{*}+\mu_{m+1: m+1: n} \sigma^{*}+w^{T} \Sigma^{-1}\left(Y-\mu^{*} \mathbf{1}-\sigma^{*} \mu\right)
$$

and its variance is given by

$$
\sigma^{2}\left\{\sigma_{m+1, m+1: m+1: n}-\omega^{T} \Sigma^{-1} \omega+\lambda_{1}^{2} 1^{T} \Sigma^{-1} 1+\lambda_{2}^{2} \mu^{T} \Sigma^{-1} \mu+2 \lambda_{1} \lambda_{2} \mu^{T} \Sigma^{-1} 1\right\},
$$

where

$$
\begin{aligned}
& Y=\left(Y_{1: m+1: n}^{\left(R_{1}, \ldots, R_{m-1}, 0, R_{m}-1\right)}, \ldots, Y_{m: m+1: n}^{\left(R_{1}, \ldots, R_{m-1}, 0, R_{m}-1\right)}\right)^{T} \\
& E(Y)=\mu 1+\sigma \mu=\left(\mu+\sigma \mu_{1: m+1: n}, \ldots, \mu+\sigma \mu_{m: m+1: n}\right)^{T} \\
& \operatorname{Var}(Y)=\sigma^{2} \Sigma=\sigma^{2}\left(\begin{array}{ccc}
\sigma_{1,1: m+1: n} & \ldots & \sigma_{1, m: m+1: n} \\
\ldots & \ldots & \ldots \\
\sigma_{m, 1: m+1: n} & \ldots & \sigma_{m, m: m+1: n}
\end{array}\right),
\end{aligned}
$$


Table 5. Variances and covariance of the BLUEs when $\mu=0$ and $\sigma=1$

\begin{tabular}{ccccccc}
\hline S.No. & $\mathrm{m}$ & $\mathrm{n}$ & $\left(R_{1}, R_{2}, \ldots, R_{m}\right)$ & $\operatorname{Var}\left(\mu^{*}\right)$ & $\operatorname{Var}\left(\sigma^{*}\right)$ & $\operatorname{Cov}\left(\mu^{*}, \sigma^{*}\right)$ \\
\hline 1 & 2 & 4 & 1,1 & 0.009165 & 1.022933 & -0.066925 \\
2 & 2 & 5 & 1,2 & 0.005791 & 1.054638 & -0.054344 \\
3 & 2 & 5 & 2,1 & 0.005781 & 1.058957 & -0.054367 \\
4 & 2 & 5 & 0,3 & 0.005781 & 1.058957 & -0.054367 \\
5 & 2 & 5 & 3,0 & 0.005541 & 0.929466 & -0.048682 \\
6 & 2 & 6 & 2,2 & 0.003967 & 1.059481 & -0.045231 \\
7 & 2 & 7 & 3,2 & 0.002883 & 1.063107 & -0.038747 \\
8 & 3 & 4 & $0,0,1$ & 0.006917 & 0.505254 & -0.032818 \\
9 & 3 & 5 & $0,0,2$ & 0.004347 & 0.525413 & -0.026700 \\
10 & 3 & 5 & $0,1,1$ & 0.004333 & 0.515230 & -0.026301 \\
11 & 3 & 5 & $2,0,0$ & 0.004250 & 0.461464 & -0.024107 \\
12 & 3 & 6 & $1,1,1$ & 0.002961 & 0.517949 & -0.021895 \\
13 & 3 & 6 & $1,2,0$ & 0.002916 & 0.485591 & -0.020685 \\
14 & 3 & 6 & $1,0,2$ & 0.002971 & 0.528151 & -0.022239 \\
15 & 3 & 7 & $0,2,2$ & 0.002150 & 0.531686 & -0.019013 \\
16 & 3 & 7 & $1,2,1$ & 0.002146 & 0.521983 & -0.018780 \\
17 & 4 & 5 & $0,0,0,1$ & 0.003864 & 0.335724 & -0.017129 \\
18 & 4 & 5 & $1,0,0,0$ & 0.003822 & 0.306044 & -0.015956 \\
19 & 4 & 6 & $1,0,1,0$ & 0.002615 & 0.321713 & -0.013656 \\
20 & 4 & 7 & $1,2,0,0$ & 0.001886 & 0.316162 & -0.011459 \\
21 & 4 & 8 & $1,1,2,0$ & 0.001434 & 0.331363 & -0.010355 \\
22 & 4 & 8 & $1,3,0,0$ & 0.002916 & 0.485591 & -0.020685 \\
23 & 4 & 8 & $2,0,0,2$ & 0.002971 & 0.528151 & -0.022239 \\
24 & 5 & 6 & $0 * 4,1$ & 0.002475 & 0.250814 & -0.010516 \\
25 & 5 & 8 & $1 * 3,0 * 2$ & 0.001345 & 0.238305 & -0.007473 \\
\hline & & & & & &
\end{tabular}

$E\left(Y_{m+1: m+1: n}^{\left(R_{1}, \ldots, R_{m-1}, 0, R_{m}-1\right)}\right)=\mu+\sigma \mu_{m+1: m+1: n}$,

$\operatorname{Var}\left(Y_{m+1: m+1: n}^{\left(R_{1}, \ldots, R_{m-1}, 0, R_{m}-1\right)}\right)=\sigma^{2} \sigma_{m+1, m+1: m+1: n}$,

$\operatorname{Cov}\left(Y_{m+1: m+1: n}^{\left(R_{1}, \ldots, R_{m-1}, 0, R_{m}-1\right)}, Y\right)=\sigma^{2} \omega=\sigma^{2}\left(\sigma_{m+1,1: m+1: n}, \ldots, \sigma_{m+1, m: m+1: n}\right)^{T}$,

$\lambda_{1}=\frac{\mu^{T} \Sigma^{-1} \mu-\mu^{T} \Sigma^{-1} \mu w^{T} \Sigma^{-1} 1-\mu_{m+1: m+1: n} \mu^{T} \Sigma^{-1} 1+\mu^{T} \Sigma^{-1} 1 w^{T} \Sigma^{-1} \mu}{\Delta}$

and

$\lambda_{2}=\frac{-\mu^{T} \Sigma^{-1} 1+\mu^{T} \Sigma^{-1} 1 w^{T} \Sigma^{-1} 1+\mu_{m+1: m+1: n} 1^{T} \Sigma^{-1} 1+1^{T} \Sigma^{-1} 1 w^{T} \Sigma^{-1} \mu}{\Delta}$

with $\Delta=\left(\mu^{T} \Sigma^{-1} \mu\right)\left(1^{T} \Sigma^{-1} 1\right)-\left(\mu^{T} \Sigma^{-1} 1\right)^{2}$. Also, $\mu_{i: m+1: n}$ and $\sigma_{i, j: m+1: n}$ denote respectively the mean and covariance of the progressively Type-II right censored order statistics from the standard $(\mu=0, \sigma=1)$ distribution, and $\mu^{*}$ and $\sigma^{*}$ are the BLUEs of $\mu$ and $\sigma$ based on the progressively Type-II censoed sample Y. The BLUPs and their variances can therefore be readily computed from the means, variances and covariances of the progressively Type-II right censoed order statistics produced in Section 5. It is also illustrated in the next section with a numerical example using a real data set.

\section{Illustrative Example}

Consider the following data which represent failure times of air conditioning equipment in a boeing 720 airplane(Proschan [23]), arranged in increasing order of magnitude: 12, 21, 26, 27, 29, 29, 48, 57, 59, 70, 74, 153, $326,386,502$. Take a random sample of size 7 from this data as: $21,26,27,29,29,48,57$ and assuming that 
this sample data follows Hjorth distribution in (6) and then producing a progressively censored data from above sample data, we have

\begin{tabular}{ccc}
\hline $\mathrm{m}$ & Scheme & $y_{i: m: n}$ \\
\hline 3 & $1,2,1$ & $21,26,29$ \\
\hline
\end{tabular}

Before carrying out the inferential analysis for these data, let us verify model assumption. Specifically, the progressively Type-II censored data $y_{i: 3: 7}$ were plotted against the values $\mu_{i: 3: 7}$ for $i=1,2,3$ determined in Section 5 (as $0.037129,0.09133,0.232938$ ) and this indicates a very high correlation (correlation coefficient is 0.922773 ) which suggest that the Hjorth model is a good fit model for these data.

In this case, we have $n=7$, and based on progressively Type-II right censored sample $y_{1: 3: 7}, y_{2: 3: 7}, y_{3: 3: 7}$ presented above, we find BLUEs of $\mu$ and $\sigma$ to be

$$
\begin{aligned}
\mu^{*} & =(1.338522 \times 21)+(-0.205897 \times 26)+(-0.132625 \times 29) \\
& =18.9095
\end{aligned}
$$

and

$$
\begin{aligned}
\sigma^{*} & =(-8.971029 \times 21)+(5.342983 \times 26)+(3.68045 \times 29) \\
& =57.258999
\end{aligned}
$$

respectively, and their standard errors to be $S E\left(\mu^{*}\right)=\sigma^{*} \sqrt{\frac{\mu^{T} \Sigma^{-1} \mu}{\delta}}=2.652661$ and $S E\left(\sigma^{*}\right)=\sigma^{*} \sqrt{\frac{1^{T} \Sigma^{-1} 1}{\delta}}=$ 41.36875765 .

We obtain the BLUP of $y_{4: 4: 7}$ to be $y_{4: 4: 7}^{*}=37.40697$ and its standard error to be $S E\left(y_{4: 4: 7}^{*}\right)=0.623606$ (by taking $w=\left(\sigma_{m+1,1: m+1: n}, \ldots, \sigma_{m+1, m: m+1: n}\right)^{T}$ and progressive censoring scheme

$\left.\left(R_{1}, \ldots, R_{m-1}, 0, R_{m}-1\right)\right)$.

\section{Conclusion}

In this paper, we have established several recurrence relations for the single and product moments of progressively Type-II right censored order statistics from Hjorth distribution. With the help of these relations and using $\mathrm{R}$ software, we have computed all the means, variances and covariances of progressively Type-II right censored order statistics for different sample sizes and all possible censoring schemes. These moments have then been used to obtain the best linear unbiased estimators (BLUEs) of location and scale parameters of location-scale Hjorth distribution (6), as well as the best linear unbiased predictors (BLUPs) of the times to failure of the surviving units in the experiment. Finally, a numerical example has been presented to illustrate all the inferential methods developed here using a real data set.

\section{Acknowledgements}

Authors are grateful to the Managing Editor and the learned referee for giving valuable comments, which led to an improvement in the presentation of the paper.

\section{REFERENCES}

1. R. Aggarwala, and N. Balakrishnan, Recurrence relations for single and product moments of progressive Type-II right censored order statistics from exponential and truncated exponential distributions, Annals of the Institute of Statistical Mathematics, vol. 48, no. 4, pp. 757-771, 1996.

2. B.C. Arnold, N. Balakrishnan, and H.N. Nagaraja, A First Course in Order Statistics, John Wiley, New York, 1992. 
3. N. Balakrishnan, Order statistics from the Half logistic distribution, Journal of Statistical Computation and Simulation , vol. 20, pp. 287-309, 1985

4. N. Balakrishnan, and R. Aggarwala, Progressive Censoring: Theory, Methods and Applications, Birkhauser, Boston, 2000.

5. N. Balakrishnan, E. K. Al-Hussaini, and H.M. Saleh, Recurrence ralations for moments of progressively censored order statistics from logistic distribution with applications to inference, Journal of Statistical Planning and Inference, vol. 141, pp. 17-30, 2011.

6. N. Balakrishnan, and A.C. Cohen, Order Statistics and Inference: Estimation Methods, San Diego: Academic Press, 1991.

7. N. Balakrishnan, and H.M. Saleh, Relations of moments of progressively Type-II censored order statistics from half-logistic distribution with applications to inference, Computational Statistics \& Data Analysis, vol. 55, pp. 2775-2792, 2011.

8. N. Balakrishnan, and H.M. Saleh, Relations for single moments of progressively Type-II censored order statistics from log-logistic distribution with applications to inference, Communications in Statistics - Theory and Methods, vol. 41, no. 5, pp. 880-906, 2012.

9. N. Balakrishnan, and H.M. Saleh, Recurrence relations for single and product moments of progressively Type-II censored order statistics from a generalized half-logistic distribution with applications to inference, Journal of Statistical Computation and Simulation, vol. 83, no. 9, pp. 1704-1721, 2013.

10. N. Balakrishnan, and H.M. Saleh, Recurrence relations for single and product moments of progressively Type-II censored order statistics from generalized logistic distribution with application to inference, Communications in Statistics - Simulation and Computation, vol. 46, no. 6, pp. 4559-4577, 2017.

11. N. Balakrishnan, and R.A. Sandhu, A simple simulation algorithm for generating progressive Type-II censored samples, The American Statistician, vol. 49, pp. 229-230, 1995.

12. A.C. Cohen, Progressively censored samples in life testing, Technometrics, vol. 5, pp. 327-329, 1963.

13. A.C. Cohen, Life testing and early failure, Technometrics, vol. 8, pp. 539-549, 1966.

14. A.C. Cohen, Multi-censored sampling in the three parameter Weibull distribution, Technometrics, vol. 17, pp. 347-351, 1975.

15. A.C. Cohen, Progressively censored sampling in the three parameter log-normal distribution, Technometrics, vol. 18, pp. 99-103, 1976.

16. A.C. Cohen, Truncated and Censored Samples. Theory and Applications, Marcel Dekker, New York, 1991.

17. A.C. Cohen, and B. J. Whitten, Parameter Estimation in Reliability and Life Span Models, Marcel Dekker, New York, 1988.

18. N. Doganaksoy, and N. Balakrishnan, A useful property of best linear unbiased predictors with applications to life-testing, The American Statistician, vol. 51, pp. 22-28, 1997.

19. U. Hjorth, Reliability distribution with increasing, decreasing, constant and bathtub-shaped failure rates, Technometrics, vol. 17, pp. 99-107, 1980.

20. M.R. Mahmoud, K.S. Sultan, and H.M. Saleh, Progressively censored data from exponential distribution: moments and estimation, Metron, vol. LXIV, no. 2, pp. 199-215, 2006.

21. N.R. Mann, Exact three-order-statistics confidence bounds on reliable life for a Weibull model with progressive censoring, Journal of the American Statistical Association, vol. 64, pp. 306-315, 1969.

22. N.R. Mann, Best linear invariant estimation for Weibull parameters under progressive censoring, Technometrics, vol. 13, pp. 521-534, 1971

23. F. Proschan, Theoretical explanation of observed decreasing failure rate, Technometrics, vol. 5, pp. 375-385, 1963.

24. N. Pushkarna, J. Saran, and R. Tiwari, L-moments and TL-moments estimation and relationships for moments of progressively type-II right censored order statistics from Frechet distribution, ProbStat Forum, vol. 8, pp. 112-122, 2015.

25. J. Saran, K. Nain, and A.P. Bhattacharya, Recurrence Relations for Single and Product Moments of Progressive Type-II Right Censored Order Statistics from Left Truncated Logistic Distribution with Application to Inference, International Journal of Mathematics and Statstics, vol. 19, no. 1, pp. 113-136, 2018.

26. J. Saran, and V. Pande, Recurrence relations for moments of progressively Type-II right censored order statistics from half-logistic distribution, Journal of Statistical Theory and Applications, vol. 11, no. 1, pp. 87-96, 2012.

27. J. Saran, and N. Pushkarna, Recurrence relations for moments of progressive Type-II right censored order statistics from Burr distribution, Statistics, A journal of Theoretical and Applied Statistics, vol. 35, no. 4, pp. 495-507, 2001

28. J. Saran, and N. Pushkarna, Moments of Progressive Type-II Right Censored Order Statistics from a General Class of Doubly Truncated Continuous Distributions, Journal of Statistical Theory and Applications, vol. 13, no. 2, pp. 162-174, 2014.

29. P.K. Sen, Progressive censoring schemes, Encyclopedia of Statistical Sciences, vol. 7, eds. S. Kotz and N.L. Johnson, Wiley, New York, pp. 296-299, 1986.

30. R. Viveros, and N. Balakrishnan, Interval estimation of parameters of life from progressively censored data, Technometrics, vol. 36, pp. 84-91, 1994. 